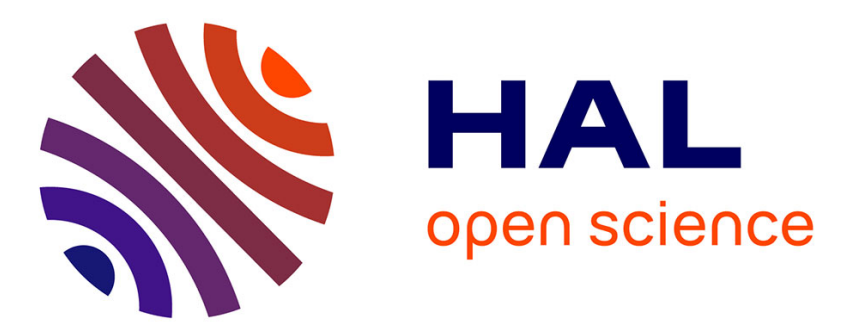

\title{
Hashin-Shtrikman bounds on the shear modulus of a nanocomposite with spherical inclusions and interface effects
}

Sébastien Brisard, Luc Dormieux, Djimedo Kondo

\section{To cite this version:}

Sébastien Brisard, Luc Dormieux, Djimedo Kondo. Hashin-Shtrikman bounds on the shear modulus of a nanocomposite with spherical inclusions and interface effects. Computational Materials Science, 2010, 50 (2), pp.403-410. 10.1016/j.commatsci.2010.08.032 . hal-00539812

\section{HAL Id: hal-00539812 \\ https://hal.science/hal-00539812}

Submitted on 4 Sep 2013

HAL is a multi-disciplinary open access archive for the deposit and dissemination of scientific research documents, whether they are published or not. The documents may come from teaching and research institutions in France or abroad, or from public or private research centers.
L'archive ouverte pluridisciplinaire HAL, est destinée au dépôt et à la diffusion de documents scientifiques de niveau recherche, publiés ou non, émanant des établissements d'enseignement et de recherche français ou étrangers, des laboratoires publics ou privés. 


\title{
Hashin-Shtrikman bounds on the shear modulus of a nanocomposite with spherical inclusions and interface effects
}

\author{
S. Brisard ${ }^{\mathrm{a}, *}$, L. Dormieux ${ }^{\mathrm{a}}$, D. Kondo \\ ${ }^{a}$ Université Paris-Est, UR Navier, Ecole des Ponts ParisTech, 6-8 av. Blaise Pascal, Cité Descartes, \\ Champs-sur-Marne, F-77455 Marne-La-Vallée cedex 2, France \\ ${ }^{b}$ Laboratoire de Mécanique de Lille, Université de Lille 1, Boulevard Paul Langevin, F-59655 Villeneuve d'Ascq, \\ France
}

\begin{abstract}
The recently developed variational framework for polarization methods in nanocomposites is applied to the determination of a lower-bound on the shear modulus of a nanocomposite with monosized, spherical inclusions. This bound explicitly accounts for linear elastic effects in the matrix-inclusion interface. Even if the polarization fields involved in its derivation are much more intricate, this bound is closely related to the classical Hashin-Shtrikman bound, with which it coincides when surface stresses are disregarded. More strikingly, when surface stresses are not disregarded, it also coincides with previously established Mori-Tanaka estimates. This result provides firm ground for the practical use of these estimates, for example for design purposes.
\end{abstract}

Key words: Nanocomposite, Surface stress, Hashin-Shtrikman bound, Spherical inclusion, Polarization

\section{Introduction}

Nanocomposites are nowadays almost routinely used in many branches of the industry; they draw their attractive properties from the high surface-to-volume ratio of the included nanoparticles. One consequence of this high ratio is that in order to achieve the same macroscopic effect, the required volume fraction of inclusions is significantly lower for nanocomposites than it is for conventional composites: this is the well-known size effect, which is partially explained by the existence of interface stresses. Indeed, for nanocomposites, the surface energy (proportional to the total surface area of the matrix-inclusion interface) becomes comparable to the bulk energy (proportional to the volume of the composite); as a consequence, surface stresses are no longer negligible.

Surface stresses cannot be accounted for by conventional homogenization schemes, and the classical micromechanics framework has recently been extended by several authors [see e.g. $1,2,3]$ to include explicitly interface effects. Thus, estimates of Mori-Tanaka type, or Generalized Self-Consistent estimates are now available for the elastic moduli of nanocomposites; as expected, these estimates show a dependence on the size of the nanoparticles.

\footnotetext{
${ }^{*}$ Corresponding author.

Email addresses: sebastien.brisard@lmsgc.enpc.fr (S. Brisard), luc.dormieux@lmsgc.enpc.fr (L. Dormieux), djimedo.kondo@univ-lille1.fr (D. Kondo) 
In homogenization theory, bounds are also an invaluable tool, since they provide rigorous constraints on the heuristically derived, previously mentioned estimates. Le Quang and He [3] have proposed upper- and lower- bounds on the elastic moduli of a composite with monosized spherical nanoparticles. These bounds generalize the classical Voigt and Reuss bounds; as such, when the contrast between the stiffnesses of the matrix, the inclusions and the interface is too high, these bounds are too loose to be of any use. Tighter bounds would then be highly desirable, and it is natural to think of a generalization of the Hashin and Shtrikman [4] bounds. However, Le Quang and He [3] observed that such a generalization would not be trivial for two reasons : $i$. the elastic stiffness of the matrix-inclusion interface might not be positive definite [5], and ii. some mathematical difficulties are raised by the singular (in the sense of generalized functions) nature of this stiffness tensor.

While $i$. might well be intractable (and following Le Quang and He [3], we will assume positive definiteness in this paper), there is a workaround to ii., which we call thin layer analogy, and introduced in a previous paper [6], in which the technique was applied to the derivation of a lower bound on the bulk modulus of a nanocomposite.

The present work is a continuation of this previous paper, in which the thin layer analogy is now used to derive a lower-bound on the shear modulus of a nanocomposite with spherical, mono-sized inclusions. It will be shown that the reasoning is more involved for the shear modulus than it was for the bulk modulus. Indeed, while the lower bound on the bulk modulus held for any homogeneous distribution of particles, isotropy will specifically be required in this paper; to account for this statistical assumption, it will prove convenient to use the framework of morphologically representative patterns proposed by Bornert et al. [7]. Furthermore, the structure of the polarization field used to get the desired bound is non-trivial, as opposed to its comparatively simple structure in the case of the bulk modulus.

It is finally proved that, provided statistical isotropy prevails, as well as positive definiteness of the stiffness of the interface, the proposed lower bound coincides with previously known Mori-Tanaka estimates [1]. This result is well-known in the context of classical composites; it is remarkable that it extends to nanocomposites.

\section{Background}

\subsection{Interface effects and the thin layer analogy}

In order to explicitly account for stress discontinuities at an interface, the classical continuity equation of the traction vector $\sigma \cdot \mathbf{n}$ (where $\mathbf{n}$ denotes the normal to the interface under consideration) must be replaced by a more general jump condition $[1,8]$

$\llbracket \boldsymbol{\sigma} \rrbracket \cdot \mathbf{n}+\left(\boldsymbol{\sigma}^{s}: \mathbf{b}\right) \mathbf{n}+\nabla^{s} \cdot \boldsymbol{\sigma}^{s}=0$

where $\nabla^{s}$ denotes the gradient operator along the interface, $\mathbf{b}$ its local curvature tensor, and $\boldsymbol{\sigma}^{s}$ the so-called surface-stress tensor. Assuming the two phases to be perfectly bound, continuity of the displacement $\mathbf{u}$ must still be enforced.

At a solid-solid interface, the values of the surface stresses are linked in a general way to the surface strains by the Shuttleworth equation [9]. Linear elasticity is a simple, physically well-founded, assumption for the stress-strain relationship of the interface, whose constitutive law then reads

$\boldsymbol{\sigma}^{s}=\mathbf{c}^{s}: \boldsymbol{\varepsilon}$, 
where $\mathbf{c}^{s}$ denotes the fourth-rank stiffness (surface) tensor of the interface; it operates on the tangential components of the (bulk) strain tensor $\varepsilon$ only.

In the three-dimensional case, thermodynamical stability of solids require their stiffness tensor to be positive definite; there is no such requirement for the stiffness of interfaces, and examples can indeed be found [5] where $\mathbf{c}^{s}$ is not positive definite. This is due to the fact that the interface has no existence on its own (it cannot be isolated from the two phases it separates), and the stability of the sole interface is not physically meaningful. However, the results presented in this paper are based on polarization methods (see below), for which positive definiteness is essential. The remainder of this paper is therefore restricted to this class of nanocomposites for which the stiffness of the interface is positive definite; more precisely, we require the bulk stiffness $\tilde{\mathbf{c}}^{s}$ of the equivalent thin layer to be positive definite, see (10).

Spherical inclusions only will be considered in this work. For such geometries, it is convenient to use spherical coordinates $(r, \theta, \varphi)$ centered at the center of the current inclusion $(\theta$ : inclination, $\varphi$ : azimut). In this system of coordinates, equilibrium of the interface (1) reads as

$\sigma_{\theta \theta}^{s}+\sigma_{\varphi \varphi}^{s}-a \llbracket \sigma_{r r} \rrbracket=0$,

$\partial_{\theta} \sigma_{\theta \theta}^{s}+\frac{1}{\sin \theta} \partial_{\varphi} \sigma_{\theta \varphi}^{s}+\left(\sigma_{\theta \theta}^{s}-\sigma_{\varphi \varphi}^{s}\right) \cot \theta+a \llbracket \sigma_{r \theta} \rrbracket=0$,

$\partial_{\theta} \sigma_{\theta \varphi}^{s}+\frac{1}{\sin \theta} \partial_{\varphi} \sigma_{\varphi \varphi}^{s}+2 \sigma_{\theta \varphi}^{s} \cot \theta+a \llbracket \sigma_{r \varphi} \rrbracket=0$,

where $\llbracket \sigma_{i j} \rrbracket=\sigma_{i j}(r=a+, \theta, \varphi)-\sigma_{i j}(r=a-, \theta, \varphi)$, and $a$ denotes the radius of the inclusion. Introducing the elastic moduli $\kappa^{s}$ and $\mu^{s}$ of the interface, constitutive equation (2) reads in spherical coordinates

$$
\begin{aligned}
& \sigma_{\theta \theta}^{s}=\left(\kappa^{s}-\mu^{s}\right)\left(\varepsilon_{\theta \theta}+\varepsilon_{\varphi \varphi}\right)+2 \mu^{s} \varepsilon_{\theta \theta}, \\
& \sigma_{\varphi \varphi}^{s}=\left(\kappa^{s}-\mu^{s}\right)\left(\varepsilon_{\theta \theta}+\varepsilon_{\varphi \varphi}\right)+2 \mu^{s} \varepsilon_{\varphi \varphi}, \\
& \sigma_{\theta \varphi}^{s}=2 \mu^{s} \varepsilon_{\theta \varphi} .
\end{aligned}
$$

It should be noted that there seems to be no agreement on the definition of the elastic coefficients of the interface; we therefore list below the substitutions to operate for the two other papers which are the most closely related to our work

- we adopted the same conventions as Le Quang and He [3]: $\kappa^{s} \rightsquigarrow \kappa_{s i}$ and $\mu^{s} \rightsquigarrow \mu_{s i}$,

- Duan et al. [1] have slightly different conventions: $\kappa^{s} \rightsquigarrow \kappa_{s} / 2$ and $\mu^{s} \rightsquigarrow \mu_{s}$.

To close this section, we now introduce a useful analogy; indeed, in some situations, it will prove convenient, even necessary, to treat the two-dimensional elastic interface as a threedimensional solid. Recognizing that jump conditions (3), (4) and (5) are in fact the equilibrium equations of a solid layer of small, but finite thickness $h$, we proved in another paper [6], that this can be done through the so-called thin layer analogy.

In this analogy, the surface stresses $\sigma^{s}$ must be identified with the product $h \sigma$, where $\sigma$ is the usual Cauchy stress tensor within the elastic layer. The constitutive (bulk) equation of this layer, consistent with the (surface) counterpart (2) then reads as

$\boldsymbol{\sigma}=\frac{\boldsymbol{\sigma}^{s}}{h}=\frac{1}{h} \mathbf{c}_{3 \mathrm{~d}}^{s}: \boldsymbol{\varepsilon}, \quad \mathbf{c}_{3 \mathrm{~d}}^{s}=2 \mu^{s}\left(\frac{2 \kappa^{s}}{3 \mu^{s}-\kappa^{s}} \mathbf{J}+\mathbf{K}\right)$, 
where $\mathbf{J}$ and $\mathbf{K}$ are the fourth rank spherical and deviatoric projectors. It should be emphasized that unlike $\mathbf{c}^{s}$, which operates on the tangential components of $\varepsilon$ only, $\mathbf{c}_{3 \mathrm{~d}}^{s}$ operates on all components of $\varepsilon$. As already mentioned, we require this stiffness to be positive definite. This leads to the following restrictions on the elastic coefficients of the interface

$0<\kappa^{s}<3 \mu^{s}$.

The thin layer analogy will be thoroughly used in section 2.3.

\subsection{Polarization-based bounds in linear elasticity}

In the present section, the main results related to variational polarization methods, the proof of which was established by Willis [10] and Ponte Castañeda and Willis [11], are exposed.

We consider a heterogeneous material occupying the domain $\Omega \subset \mathbb{R}^{3}$, with local elastic stiffness $\mathbf{c}(\mathbf{x})$. The derivation of the effective stiffness $\mathbf{C}$ of this medium requires the resolution of the following micromechanics problem

$\operatorname{div}[\mathbf{c}(\mathbf{x}): \boldsymbol{\varepsilon}(\mathbf{x})]=0 \quad(\mathbf{x} \in \Omega)$,

$\mathbf{u}(\mathbf{x})=\mathbf{E} \cdot \mathbf{x} \quad(\mathbf{x} \in \partial \Omega)$

where $\mathbf{E}$ is the macroscopic strain, $\mathbf{u}$ the local displacement, and $\boldsymbol{\varepsilon}$ the corresponding local strain, $\varepsilon_{i j}=\left(\partial_{i} u_{j}+\partial_{j} u_{i}\right) / 2$. The macroscopic stiffness $\mathbf{C}$ is then retrieved from the averaging rule

$\mathbf{C}: \mathbf{E}=\overline{\mathbf{c}(\mathbf{x}): \varepsilon(\mathbf{x})}$,

where overlined quantities denote volume averages

$\overline{\mathcal{B}}=\frac{1}{|\Omega|} \int_{\mathbf{x} \in \Omega} \mathcal{B}(\mathbf{x}) \mathrm{d}^{3} \mathbf{x}$.

A fruitful approach to the preceding problem consists of introducing a so-called reference medium with homogeneous elastic stiffness $\mathbf{c}^{0}$, occupying the same domain $\Omega$. Equation (11) can then be replaced by the following equilibrium equation, written on the homogeneous, prestressed reference medium

$\operatorname{div}\left[\mathbf{c}^{0}: \boldsymbol{\varepsilon}(\mathbf{x})+\boldsymbol{\tau}(\mathbf{x})\right]=0 \quad(\mathbf{x} \in \Omega)$,

where the so-called polarization field $\tau(\mathbf{x})$ has been introduced

$\boldsymbol{\tau}=\left[\mathbf{c}(\mathbf{x})-\mathbf{c}^{0}\right]: \boldsymbol{\varepsilon}(\mathbf{x})$.

It should be noted that the whole complexity of the original micromechanics problem, arising from the heterogeneity of $\mathbf{c}(\mathbf{x})$, has been transfered to the polarization field $\tau(\mathbf{x})$. If this field were known, the solution of the polarized problem would read

$\varepsilon(\mathbf{x})=\mathbf{E}-\left(\Gamma^{0} \circledast \tau\right)(\mathbf{x})$,

where $\Gamma^{0}$ stands for the two-point, fourth-rank Green tensor for strains [12], and ' $\circledast$ ' denotes its product with a one point, second rank tensor

$\left(\boldsymbol{\Gamma}^{0} \circledast \tau\right)(\mathbf{x})=\int_{\mathbf{y} \in \Omega} \Gamma^{0}(\mathbf{x}, \mathbf{y}): \tau(\mathbf{y}) \mathrm{d}^{3} \mathbf{y}$. 
The polarization field is in general not known, but (17) is still valid, and $\tau(\mathbf{x})$ solves the following implicit equation

$\tau(\mathbf{x})+\left[\mathbf{c}(\mathbf{x})-\mathbf{c}^{0}\right]:\left(\Gamma^{0} \circledast \tau\right)(\mathbf{x})=\left[\mathbf{c}(\mathbf{x})-\mathbf{c}^{0}\right]: \mathbf{E}$,

where it is noted that equation (19) is self-standing, all boundary conditions being 'included' in the Green operator for strains itself. The effective stiffness of the original heterogeneous medium then follows from

$\mathbf{C}: \mathbf{E}=\mathbf{c}^{0}: \mathbf{E}+\overline{\boldsymbol{\tau}}$

The variational counterpart of equation (19) has been derived by Willis [10]. It states that for any choice of the polarization field $\tau(\mathbf{x})$,

$$
\begin{aligned}
\frac{1}{2} \mathbf{E}: \mathbf{C}: \mathbf{E} \geq & \frac{1}{2} \mathbf{E}: \mathbf{c}^{0}: \mathbf{E}+\overline{\boldsymbol{\tau}}: \mathbf{E} \\
& -\frac{1}{2} \overline{\boldsymbol{\tau}:\left(\mathbf{c}-\mathbf{c}^{0}\right)^{-1}: \boldsymbol{\tau}}-\frac{1}{2} \overline{\boldsymbol{\tau}:\left(\boldsymbol{\Gamma}^{0} \circledast \boldsymbol{\tau}\right)} .
\end{aligned}
$$

For this inequality to hold, the reference medium must be softer than any of the phases in the original composite ; in other words, $\mathbf{c}(\mathbf{x})-\mathbf{c}^{0}$ must be positive definite for all $\mathbf{x} \in \Omega$ (this inequality is reversed if the reference medium is stiffer than any of the phases in the original composite, that is $\mathbf{c}(\mathbf{x})-\mathbf{c}^{0}$ is negative definite for all $\left.\mathbf{x} \in \Omega\right)$. It should be emphasized that no constraint is imposed on the polarization field $\tau$, therefore, any choice of $\tau$ leads to a bound on the macroscopic elastic energy.

As was proved by Willis [10] and Ponte Castañeda and Willis [11], the classical bounds of Hashin and Shtrikman [4] are retrieved by selecting piecewise constant polarization fields. In the present work, we propose carefully selected, non-trivial, polarization fields leading to bounds on the shear modulus of nanocomposites. However, the classical variational framework cannot handle interface effects, and new extensions of this framework, based on the thin layer analogy, will first be proposed in section 2.3 [see also 6].

\subsection{Morphologically representative pattern approach}

When no reference is made to the topological nature of the phases of a composite, the only natural choice for the polarization field $\tau(\mathbf{x})$ is a constant by phase field. This is indeed the choice done by Willis [10], who then produced a new derivation of the bounds previously obtained by Hashin and Shtrikman [4]. Intuitively, it is realized that these bounds could be improved on by selecting non-constant polarization fields. However, resorting to locally variable polarization fields means that each single point of the heterogeneous medium is handled specifically. This must be done carefully in order to comply with the assumed global statistical homogeneity of the medium. Otherwise, the quality of the resulting bounds will turn out to be very poor.

The task is somewhat easier with particulate composites, for which the centers of each particle can be used as reference points, without breaking the statistical homogeneity. Making use of constant polarization fields, Ponte Castañeda and Willis [11] have already proposed HashinShtrikman type bounds for particulate composites made of ellipsoidal inclusions whose distribution presents an ellipsoidal symmetry. Bornert et al. [7] have then extended this approach to locally variable polarization fields, which they called morphologically representative patterns. This latter approach is used in the present work. 
For the sake of completeness, we summarize here the main results obtained in [7]. Following these authors, we call "pattern" a bounded (possibly heterogenous) subdomain of a composite. If the composite can be retrieved from copies (by translation) of a finite number of such patterns, these will be coined morphologically representative. For example, the nanocomposites considered below can be retrieved from copies of the association of the elastic inclusion with the elastic interface

pattern $=$ inclusion + interface.

We consider a distribution of $N$ identical patterns (superscript ' $p$ ') embedded in a matrix (superscript ' $m$ ', stiffness $\left.\mathbf{c}^{m}\right)$. Pattern $\alpha(\alpha=1, \ldots, N)$ is centered at point $\mathbf{x}^{\alpha}$, and $\mathbf{x} \mapsto \chi^{p}(\mathbf{x})$ denotes the characteristic function of the reference pattern, centered at the origin, so that $\mathbf{x} \mapsto$ $\chi^{p}\left(\mathbf{x}-\mathbf{x}^{\alpha}\right)$ is the characteristic function of pattern $\alpha$. The pattern can be heterogeneous, and $\mathbf{x} \mapsto \mathbf{c}^{p}(\mathbf{x})$ denotes the local stiffness of the reference pattern $\left(\mathbf{c}^{p}(\mathbf{x})=\mathbf{0}\right.$ if $\left.\chi^{p}(\mathbf{x})=0\right)$. The stiffness at point $\mathbf{x} \in \Omega$ therefore reads

$\mathbf{c}(\mathbf{x})=\mathbf{c}^{m}+\sum_{\alpha=1}^{N}\left[\mathbf{c}^{p}\left(\mathbf{x}-\mathbf{x}^{\alpha}\right)-\chi^{p}\left(\mathbf{x}-\mathbf{x}^{\alpha}\right) \mathbf{c}^{m}\right]$.

The reference medium $\mathbf{c}^{0}$ in (21) is yet unspecified, and we assume the general form for the polarization field $\tau(\mathbf{x})$

$\boldsymbol{\tau}(\mathbf{x})=\sum_{\alpha=1}^{N} \tau^{p}\left(\mathbf{x}-\mathbf{x}^{\alpha}\right)$

where it is understood that $\boldsymbol{\tau}^{p}(\mathbf{x})=\mathbf{0}$ if $\chi^{p}(\mathbf{x})=0$. In other words, each copy of the morphologically representative pattern is submitted to the same (locally variable) polarization field, while the matrix is intentionally not polarized. It will be convenient to introduce the following volume average

$\overline{\boldsymbol{\tau}}^{p}=\frac{1}{\left|\Omega^{p}\right|} \int_{\mathbf{x} \in \Omega^{p}} \boldsymbol{\tau}^{p}(\mathbf{x}) \mathrm{d}^{3} x$,

where $\Omega^{p} \subset \mathbb{R}^{3}$ denotes the domain occupied by the reference pattern, centered at the origin, $\Omega^{p}=\chi^{p-1}(\{1\})$.

Introducing (23) in the general variational inequality (21) then leads to a bound on the macroscopic potential energy of the composite. Bornert et al. [7] have already observed that the socalled interaction term, namely

$\overline{\tau:\left(\Gamma^{0} \circledast \tau\right)}=\frac{1}{V} \int_{\mathbf{x} \in \Omega} \tau(\mathbf{x}):\left(\Gamma^{0} \circledast \tau\right)(\mathbf{x}) \mathrm{d}^{3} \mathbf{x}$,

depends explicitly on the distribution of the centers of the patterns; it leads to volume integrals which are in general intractable. However, adopting a statistical approach, in which many realizations of the same random heterogeneous material are considered, leads to almost explicit expressions for the interaction term. Indeed, inequality (21) holds for any realization, and ensemble averages (denoted by $\langle\cdot\rangle)$ can be taken on both sides

$$
\begin{aligned}
\frac{1}{2} \mathbf{E}:\langle\mathbf{C}\rangle: \mathbf{E} \geq & \frac{1}{2} \mathbf{E}: \mathbf{c}^{0}: \mathbf{E}+\langle\overline{\boldsymbol{\tau}}\rangle: \mathbf{E}-\frac{1}{2}\left\langle\overline{\left.\boldsymbol{\tau}:\left(\mathbf{c}-\mathbf{c}^{0}\right)^{-1}: \boldsymbol{\tau}\right\rangle}\right. \\
& \left.-\frac{1}{2} \overline{\left\langle\boldsymbol{\tau}:\left(\boldsymbol{\Gamma}^{0} \circledast \boldsymbol{\tau}\right)\right.}\right\rangle .
\end{aligned}
$$


The analysis is further simplified by adopting the same local polarization field $\tau^{p}$ for each realization. The random nature of the global polarization field $\tau$ is therefore only due to the distribution of centers $\mathbf{x}^{\alpha}, \alpha=1, \ldots, N$. Assuming these centers to be distributed isotropically, the results proved by Bornert et al. [7] for an ellipsoidal distribution apply, and

$$
\begin{aligned}
& \left\langle\overline{\boldsymbol{\tau}:\left(\boldsymbol{\Gamma}^{0} \circledast \boldsymbol{\tau}\right)}\right\rangle= \\
& \quad \frac{f}{\left|\Omega^{p}\right|} \int_{\mathbf{x}, \mathbf{y} \in \Omega^{p}} \boldsymbol{\tau}^{p}(\mathbf{x}): \boldsymbol{\Gamma}_{\infty}^{0}(\mathbf{x}-\mathbf{y}): \boldsymbol{\tau}^{p}(\mathbf{y}) \mathrm{d}^{3} \mathbf{x} \mathrm{d}^{3} \mathbf{y} \\
& \quad-f^{2} \overline{\boldsymbol{\tau}}^{p}: \mathbf{P}^{\mathrm{sph}}: \overline{\boldsymbol{\tau}}^{p},
\end{aligned}
$$

where $f=N\left|\Omega^{p}\right| /|\Omega|$ denotes the volume fraction of the patterns, and $\mathbf{P}^{\text {sph }}$ the Hill tensor of a sphere. It should be noted that the previous result is obtained in the thermodynamic limit $\left(|\Omega| \rightarrow+\infty, N /|\Omega|=\right.$ const.), in which case the finite-body Green operator $\boldsymbol{\Gamma}^{0}$ operating on a polarization field $\tau$ may be replaced by the infinite-body Green operator $\boldsymbol{\Gamma}_{\infty}^{0}$, operating on the modified polarization field $\tau-\bar{\tau}[10]$

$$
\left(\boldsymbol{\Gamma}^{0} \circledast \boldsymbol{\tau}\right)(\mathbf{x}) \simeq \int_{\mathbf{y} \in \Omega} \boldsymbol{\Gamma}_{\infty}^{0}(\mathbf{x}-\mathbf{y}):[\boldsymbol{\tau}(\mathbf{y})-\bar{\tau}] \mathrm{d}^{3} \mathbf{y} .
$$

The first term on the right-hand side of (27) involves only one copy of the pattern; it will be called the self-influence term. That the interaction term contains only intra-pattern interactions (no inter-pattern interaction) when ellipsoidal symmetry prevails is the remarkable result proved by Bornert et al. [7]. Paramount in the present work is the observation that the strain field $\boldsymbol{\varepsilon}^{p}(\mathbf{x})$, defined as

$\boldsymbol{\varepsilon}^{p}(\mathbf{x})=-\int_{\mathbf{y} \in \Omega^{p}} \Gamma_{\infty}^{0}(\mathbf{x}-\mathbf{y}): \boldsymbol{\tau}^{p}(\mathbf{y}) \mathrm{d}^{3} \mathbf{y}$,

is the solution to the generalized inhomogeneity problem

$$
\begin{array}{ll}
\operatorname{div}\left[\mathbf{c}^{m}: \boldsymbol{\varepsilon}^{p}(\mathbf{x})+\boldsymbol{\tau}^{p}(\mathbf{x})\right]=0 & \left(\mathbf{x} \in \mathbb{R}^{3}\right), \\
\mathbf{u}^{p}(\mathbf{x}) \rightarrow 0 & (|\mathbf{x}| \rightarrow+\infty),
\end{array}
$$

where $\mathbf{u}^{p}(\mathbf{x})$ is the displacement associated with the strain field $\boldsymbol{\varepsilon}^{p}(\mathbf{x})$. The self-influence term therefore reads

$-\frac{1}{\left|\Omega^{p}\right|} \int_{\mathbf{x} \in \Omega^{p}} \tau^{p}(\mathbf{x}): \boldsymbol{\varepsilon}^{p}(\mathbf{x}) \mathrm{d}^{3} \mathbf{x}$.

The remaining terms in (26) are readily expressed as a function of the local polarization field $\tau^{p}(\mathbf{x})$

$\langle\bar{\tau}\rangle=f \overline{\boldsymbol{\tau}}^{p}$,

and

$$
\begin{aligned}
& \left.\overline{\left\langle\boldsymbol{\tau}:\left(\mathbf{c}-\mathbf{c}^{0}\right)^{-1}: \boldsymbol{\tau}\right.}\right\rangle= \\
& \quad \frac{f}{\left|\Omega^{p}\right|} \int_{\mathbf{x} \in \Omega^{p}} \boldsymbol{\tau}^{p}(\mathbf{x}):\left[\mathbf{c}^{p}(\mathbf{x})-\mathbf{c}^{0}\right]^{-1}: \boldsymbol{\tau}^{p}(\mathbf{x}) \mathrm{d}^{3} \mathbf{x} .
\end{aligned}
$$


Inserting (27), (32), (33) and (34) into (26) results in the following bound on the macroscopic energy

$$
\begin{aligned}
& \frac{1}{2} \mathbf{E}:\langle\mathbf{C}\rangle: \mathbf{E} \geq \frac{1}{2} \mathbf{E}: \mathbf{c}^{0}: \mathbf{E}+f \bar{\tau}^{p}: \mathbf{E} \\
& \quad-\frac{f}{2\left|\Omega^{p}\right|} \int_{\mathbf{x} \in \Omega^{p}} \tau^{p}(\mathbf{x}):\left[\mathbf{c}^{p}(\mathbf{x})-\mathbf{c}^{0}\right]^{-1}: \tau^{p}(\mathbf{x}) \mathrm{d}^{3} \mathbf{x} \\
&+\frac{f}{2\left|\Omega^{p}\right|} \int_{\mathbf{x} \in \Omega^{p}} \tau^{p}(\mathbf{x}): \boldsymbol{\varepsilon}^{p}(\mathbf{x}) \mathrm{d}^{3} \mathbf{x}+\frac{f^{2}}{2} \bar{\tau}^{p}: \mathbf{P}^{\mathrm{sph}}: \bar{\tau}^{p},
\end{aligned}
$$

which holds for any choice of the macroscopic strain tensor $\mathbf{E}$, and the local polarization field $\tau^{p}(\mathbf{x})$. In the next section, specific choices of these parameters will allow for the derivation of a bound on the shear modulus of a nanocomposite.

\section{Hashin-Shtrikman-like bounds for nanocomposites}

\subsection{Polarization methods in the presence of interface effects}

In the present section we derive, within the framework presented above, bounds on the elasticity of nanocomposites; these bounds take explicitly into account interface effects.

We consider here an isotropic distribution of spherical homogeneous inclusions (superscript ' $i$ ', stiffness $\mathbf{c}^{i}$, bulk modulus $\kappa^{i}$, shear modulus $\mu^{i}$, radius $a$ ) embedded in a matrix (superscript ' $m$ ', stiffness $\mathbf{c}^{m}$, bulk modulus $\kappa^{m}$, shear modulus $\mu^{m}$ ). Surface stresses are allowed to develop at the matrix/inclusion boundary, and the interface (superscript ' $s$ ') is assumed to behave linearly, with (2d) stiffness $\mathbf{c}^{s}$. It is further assumed that the elastic coefficients of the interface verify (10), which, according to section 1.1, permits the use of an equivalent thin elastic layer with positive definite stiffness $\mathbf{c}_{3 \mathrm{~d}}^{s} / h$, see (9).

In order to act as reinforcement, the inclusions must be stiffer than the embedding matrix. Besides, the thickness $h$ of the equivalent thin elastic layer is arbitrarily small, and the corresponding fictitious elastic material arbitrarily stiff. In other words, the matrix is softer than both the inclusions and the interface. It can therefore be chosen as the reference medium, and the use of polarization methods will then lead to lower bounds on the elastic properties of the composite.

Before we proceed, it should be noted that for such a choice of the reference medium, the polarization field must vanish within the matrix. This is due to the fact that in (21), the term

$$
\frac{1}{2} \overline{\tau:\left(\mathbf{c}-\mathbf{c}^{0}\right)^{-1}: \tau}=\frac{1}{2} \overline{\tau:\left(\mathbf{c}-\mathbf{c}^{m}\right)^{-1}: \tau}
$$

is singular when $\mathbf{x}$ belongs to the matrix. This remark is consistent with (23), where the polarization field was non-zero inside the copies of the pattern only. As already mentioned above, the morphologically representative pattern considered here is the association of the inclusion and the interface, which leads to a decomposition of the local polarization field as the sum of a $3 \mathrm{~d}$ and a $2 \mathrm{~d}$ term

$\tau^{p}(\mathbf{x})=\tau^{i}(\mathbf{x})+\delta(r-a) \tau^{s}(\mathbf{n})$,

where $a$ is the radius of the spherical inclusions, $r=|\mathbf{x}|$ and $\mathbf{n}=\mathbf{x} / r$; furthermore, $\tau^{i}(\mathbf{x})=0$ for $r>a$. The precise expression of $\tau^{i}$ and $\tau^{s}$ is deferred to section 2.2. From the definition (37), it readily follows that

$\overline{\boldsymbol{\tau}}^{p}=\overline{\boldsymbol{\tau}}^{i}+\frac{3}{a} \overline{\boldsymbol{\tau}}^{s}$, 
where the following volume (resp. angular) average of $\tau^{i}$ (resp. $\tau^{s}$ ) have been introduced

$\overline{\boldsymbol{\tau}}^{i}=\frac{1}{\left|\Omega^{p}\right|} \int_{\mathbf{x} \in \Omega^{p}} \tau^{i}(\mathbf{x}) \mathrm{d}^{3} \mathbf{x}, \quad \overline{\boldsymbol{\tau}}^{s}=\frac{1}{4 \pi} \int_{|\mathbf{n}|=1} \tau^{s}(\mathbf{n}) \mathrm{d}^{2} \mathbf{n}$,

with $\Omega^{p}=\{\mathbf{x},|\mathbf{x}| \leq a\}$, and $\left|\Omega^{p}\right|=4 \pi a^{3} / 3$.

Aiming at evaluating the bound (35) resulting from (37), we observe that the singular nature of the interface raises two issues which we address below.

Firstly, evaluation of the self-influence term, as expressed in (27) might seem impossible, since both $\tau^{p}(\mathbf{x})$ and $\tau^{p}(\mathbf{y})$ are singular fields. However, the strain field $\boldsymbol{\varepsilon}^{p}$ induced by $\boldsymbol{\tau}^{p}$ is regular, and expression (32) of the self-influence term should therefore be used. Besides, the inter-pattern interaction term, which was null for regular local polarization fields, remains null for $\tau^{p}$ given by (37). To realize this, we can use the thin elastic layer analogy developed in section 1.1. Then the singular part $\delta(r-a) \tau^{s}(\mathbf{n})$ of the local polarization field is replaced by a regular field, defined for $a \leq r \leq a+h$ by $\tau^{s}(\mathbf{n}) / h$. Using then the results proved by Bornert et al. [7], it is seen that the inter-pattern interaction term is null for any choice of the thickness $h$. Taking the limit $h \rightarrow 0$ proves that this remains true for the singular local polarization field selected here. The interaction term (27) therefore reads

$$
\begin{aligned}
\left\langle\overline{\boldsymbol{\tau}:\left(\boldsymbol{\Gamma}^{0} \circledast \boldsymbol{\tau}\right)}\right\rangle=-\frac{f}{\left|\Omega^{p}\right|} \int_{\mathbf{x} \in \Omega^{p}} \boldsymbol{\tau}^{i}(\mathbf{x}): & \boldsymbol{\varepsilon}^{p}(\mathbf{x}) \mathrm{d}^{3} \mathbf{x} \\
& -\frac{f a^{2}}{\left|\Omega^{p}\right|} \int_{|\mathbf{n}|=1} \boldsymbol{\tau}^{s}(\mathbf{n}): \boldsymbol{\varepsilon}^{p}(a \mathbf{n}) \mathrm{d}^{2} \mathbf{n}-f^{2} \overline{\boldsymbol{\tau}}^{p}: \mathbf{P}^{\mathrm{sph}}: \overline{\boldsymbol{\tau}}^{p} .
\end{aligned}
$$

Secondly, evaluation of the term in (35) involving the difference $\mathbf{c}^{p}(\mathbf{x})-\mathbf{c}^{0}$ is pursued. In this term simultaneously appear the stiffness of the interface (surface tensor) and that of the reference material (volume tensor). These two mathematical entities are distinct in nature and cannot be compared without further treatment. Again, the thin elastic layer analogy introduced in section 1.1 suggests to replace the surface integral stemming from the $\delta(r-a)$ term by a volume integral on a thin layer $a \leq r \leq a+h(h \ll a)$. Then $\mathbf{c}_{3 \mathrm{~d}}^{s} / h$ is substituted to $\mathbf{c}^{s}$, and $\tau^{s} / h$ to $\tau^{s}$

$$
\begin{aligned}
& \int_{\mathbf{x} \in \Omega^{p}} \boldsymbol{\tau}^{p}(\mathbf{x}):\left[\mathbf{c}^{p}(\mathbf{x})-\mathbf{c}^{m}\right]^{-1}: \boldsymbol{\tau}^{p}(\mathbf{x}) \mathrm{d}^{3} \mathbf{x}= \\
& \quad+\int_{a}^{a+h} r^{3} \mathrm{~d} r \int_{|\mathbf{n}|=1} \frac{\boldsymbol{\tau}^{s}(\mathbf{n})}{h}:\left(\mathbf{c}_{3 \mathrm{~d}}^{s} / h-\mathbf{c}^{m}\right)^{-1}: \frac{\boldsymbol{\tau}^{s}(\mathbf{n})}{h} \mathrm{~d}^{3} \mathbf{x} \\
& \quad+\int_{\mathbf{x} \in \Omega^{p}} \boldsymbol{\tau}^{i}(\mathbf{x}):\left(\mathbf{c}^{i}-\mathbf{c}^{m}\right)^{-1}: \boldsymbol{\tau}^{i}(\mathbf{x}) \mathrm{d}^{3} \mathbf{x} .
\end{aligned}
$$

Radial integration in the thin layer is readily performed, since $h \ll a$; besides, the following asymptotic behavior holds

$$
\left(\mathbf{c}_{3 \mathrm{~d}}^{s} / h-\mathbf{c}^{m}\right)^{-1} \sim h\left(\mathbf{c}_{3 \mathrm{~d}}^{s}\right)^{-1} \quad(h \rightarrow 0),
$$


therefore, in the limit $h \rightarrow 0$, we find

$$
\begin{array}{r}
\int_{\mathbf{x} \in \Omega^{p}} \boldsymbol{\tau}^{p}(\mathbf{x}):\left[\mathbf{c}^{p}(\mathbf{x})-\mathbf{c}^{m}\right]^{-1}: \boldsymbol{\tau}^{p}(\mathbf{x}) \mathrm{d}^{3} \mathbf{x}= \\
\int_{\mathbf{x} \in \Omega^{p}} \boldsymbol{\tau}^{i}(\mathbf{x}):\left(\mathbf{c}^{i}-\mathbf{c}^{m}\right)^{-1}: \boldsymbol{\tau}^{i}(\mathbf{x}) \mathrm{d}^{3} \mathbf{x} \\
\quad+a^{2} \int_{|\mathbf{n}|=1} \boldsymbol{\tau}^{s}(\mathbf{n}):\left(\mathbf{c}_{3 \mathrm{~d}}^{s}\right)^{-1}: \boldsymbol{\tau}^{s}(\mathbf{n}) \mathrm{d}^{2} \mathbf{n} .
\end{array}
$$

Introducing (38), (40) and (42) in (35), we obtain the following bound, which holds for any choice of the macroscopic strain $\mathbf{E}$ and local polarization fields $\tau^{i}$ and $\tau^{s}$

$$
\begin{aligned}
\frac{1}{2} \mathbf{E}:\langle\mathbf{C}\rangle: \mathbf{E} \geq & \frac{1}{2} \mathbf{E}: \mathbf{c}^{m}: \mathbf{E}+f\left(\overline{\boldsymbol{\tau}}^{i}+\frac{3}{a} \overline{\boldsymbol{\tau}}^{s}\right): \mathbf{E} \\
& -\frac{f}{2\left|\Omega^{p}\right|} \int_{\mathbf{x} \in \Omega^{p}} \tau^{i}(\mathbf{x}):\left(\mathbf{c}^{i}-\mathbf{c}^{m}\right)^{-1}: \boldsymbol{\tau}^{i}(\mathbf{x}) \mathrm{d}^{3} \mathbf{x} \\
& -\frac{f a^{2}}{2\left|\Omega^{p}\right|} \int_{|\mathbf{n}|=1} \tau^{s}(\mathbf{n}):\left(\mathbf{c}_{3 \mathrm{~d}}^{s}\right)^{-1}: \boldsymbol{\tau}^{s}(\mathbf{n}) \mathrm{d}^{2} \mathbf{n} \\
& +\frac{f}{2\left|\Omega^{p}\right|} \int_{\mathbf{x} \in \Omega^{p}} \tau^{i}(\mathbf{x}): \boldsymbol{\varepsilon}^{p}(\mathbf{x}) \mathrm{d}^{3} \mathbf{x} \\
& +\frac{f a^{2}}{2\left|\Omega^{p}\right|} \int_{|\mathbf{n}|=1} \boldsymbol{\tau}^{s}(\mathbf{n}): \boldsymbol{\varepsilon}^{p}(a \mathbf{n}) \mathrm{d}^{2} \mathbf{n} \\
& -f^{2}\left(\overline{\boldsymbol{\tau}}^{i}+\frac{3}{a} \overline{\boldsymbol{\tau}}^{s}\right): \mathbf{P}^{\mathrm{sph}}:\left(\overline{\boldsymbol{\tau}}^{i}+\frac{3}{a} \overline{\boldsymbol{\tau}}^{s}\right)
\end{aligned}
$$

It generalizes the framework developed in $[7,10,11]$ to composites with interface effects. In the remainder of this section, appropriate choices for the local polarization fields $\tau^{i}$ and $\tau^{s}$ will be made, in order to derive explicit bounds on the macroscopic shear modulus of the nanocomposite.

It should be mentioned that in [6], a lower bound on the bulk modulus of the nanocomposite considered here was obtained with the following, comparatively simple polarization fields

$\tau^{i}(\mathbf{x})=\tau^{i} \mathbf{i}, \quad \tau^{s}(\mathbf{n})=\tau^{s}(\mathbf{i}-\mathbf{n} \otimes \mathbf{n})$,

where $\tau^{i}$ and $\tau^{s}$ are two free scalar parameters, and $\mathbf{i}$ is the second-rank identity tensor. The effective shear modulus, which is the topic of the present work, requires to resort to more complex polarization fields.

\subsection{Specification of the macroscopic strain and the local polarization field}

In this section, we select a purely deviatoric macroscopic strain $\mathbf{E}$, together with a non-trivial local polarization field $\tau^{p}$ depending on four dimensionless constants $S, T, U$, and $V$.

Macroscopic strain. In order to derive a bound involving the macroscopic shear modulus only, the following macroscopic strain must obviously be selected

$\mathbf{E}=E\left(\mathbf{e}_{x} \otimes \mathbf{e}_{y}+\mathbf{e}_{y} \otimes \mathbf{e}_{x}\right)$,

where $E$ is an arbitrary constant. We then have

$\frac{1}{2} \mathbf{E}:\langle\mathbf{C}\rangle: \mathbf{E}=2\langle G\rangle E^{2}, \quad \frac{1}{2} \mathbf{E}: \mathbf{c}^{m}: \mathbf{E}=2 \mu^{m} E^{2}$,

where $\langle G\rangle$ stands for the effective shear modulus of the composite. 
Polarization of the inclusion. The polarization field $\tau^{i}$ used in this paper is defined in terms of the well-known solution for a sheared sphere [13], the displacements of which are recalled below

$$
\begin{aligned}
\mathcal{U}_{r}[v, A, B, C, D](r, \theta, \varphi)= & \left(A r-\frac{6 v}{1-2 v} B r^{3}+\frac{3 C}{r^{4}}\right. \\
& \left.+\frac{5-4 v}{1-2 v} \frac{D}{r^{2}}\right) \sin ^{2} \theta \sin 2 \varphi, \\
\mathcal{U}_{\theta}[v, A, B, C, D](r, \theta, \varphi)= & \left(A r-\frac{7-4 v}{1-2 v} B r^{3}-\frac{2 C}{r^{4}}\right. \\
& \left.+\frac{2 D}{r^{2}}\right) \sin \theta \cos \theta \sin 2 \varphi, \\
\mathcal{U}_{\varphi}[v, A, B, C, D](r, \theta, \varphi)= & \left(A r-\frac{7-4 v}{1-2 v} B r^{3}-\frac{2 C}{r^{4}}\right. \\
& \left.+\frac{2 D}{r^{2}}\right) \sin \theta \cos 2 \varphi,
\end{aligned}
$$

where $A, B, C$ and $D$ are four integration constants, and $v$ stands for the Poisson ratio of the material under consideration. Denoting $\mathcal{E}[v, A, B, C, D]$ the strain field derived from $\mathcal{U}$, it can be shown that

$\operatorname{div}(\mathbf{c}: \mathcal{E}[v, A, B, C, D])=0$

where $\mathbf{c}$ stands for the stiffness tensor of any linearly elastic (isotropic) material, with Poisson ratio $v$. The derivation of the bound proposed in this paper relies heavily on property (49), where attention should be paid to the fact that the value of the Poisson ratio to be inserted in the expression of $\mathcal{E}$ derived from (46), (47) and (48) is equal to that of the material whith stiffness $\mathbf{c}$. The following averaging rule can also be retrieved, and will be used below [see e.g. 14]

$$
\begin{aligned}
\frac{3}{4 \pi\left(r_{2}^{3}-r_{1}^{3}\right)} \int_{r_{1} \leq|\mathbf{x}| \leq r_{2}} \mathcal{E}[v, A, B, C, D](\mathbf{x}) \mathrm{d}^{3} \mathbf{x}= & \\
& {\left[A-\frac{21}{5} \frac{r_{2}^{5}-r_{1}^{5}}{(1-2 v)\left(r_{2}^{3}-r_{1}^{3}\right)} B\right]\left(\mathbf{e}_{x} \otimes \mathbf{e}_{y}+\mathbf{e}_{y} \otimes \mathbf{e}_{x}\right) . }
\end{aligned}
$$

$S$ and $T$ being two arbitrary, dimensionless constants, we now define the volumic part $\tau^{i}$ of the local polarization field $\tau^{p}$

$$
\boldsymbol{\tau}^{i}(\mathbf{x})=\chi^{p}(\mathbf{x})\left(\mathbf{c}^{i}-\mathbf{c}^{m}\right): \mathcal{E}\left[v^{i}, S, T / a^{2}, 0,0\right](\mathbf{x}),
$$

where $v^{i}$ refers to the Poisson ratio of the inclusions. Substituting $r_{1}=0$ and $r_{2}=a$ in (50), the volume average of $\tau^{i}$ is found

$\overline{\boldsymbol{\tau}}^{i}=2\left(\mu^{i}-\mu^{m}\right)\left(S-\frac{21}{5} \frac{T}{1-2 v^{i}}\right)\left(\mathbf{e}_{x} \otimes \mathbf{e}_{y}+\mathbf{e}_{y} \otimes \mathbf{e}_{x}\right)$.

Polarization of the interface. The choice of the local polarization field $\tau^{s}$ was suggested by the solution to Eshelby's problem for a single spherical particle with interface effects; this solution 
is not reproduced here. $U$ and $V$ being two arbitrary, dimensionless, constants, the components of $\tau^{s}$ in spherical coordinates read

$$
\begin{aligned}
\tau_{\theta \theta}^{s}= & \mu^{i} a[\sin (2 \theta+2 \varphi)-\sin (2 \theta-2 \varphi)](U+V) \\
& -2 \mu^{i} a \sin 2 \varphi(U-3 V), \\
\tau_{\varphi \varphi}^{s}= & \mu^{i} a[\sin (2 \theta+2 \varphi)-\sin (2 \theta-2 \varphi)](U-V) \\
& -2 \mu^{i} a \sin 2 \varphi(U+3 V), \\
\tau_{\theta \varphi}^{s}= & 4 \mu^{i} a[\cos (\theta+2 \varphi)+\cos (\theta-2 \varphi)] V,
\end{aligned}
$$

from which the angular average $\bar{\tau}^{s}$ is easily found

$\overline{\boldsymbol{\tau}}^{s}=\frac{8}{15} a \mu^{i}(U+6 V)\left(\mathbf{e}_{x} \otimes \mathbf{e}_{y}+\mathbf{e}_{y} \otimes \mathbf{e}_{x}\right)$,

as well as the surface divergence

$$
\begin{aligned}
\left(\boldsymbol{\tau}^{s}: \mathbf{b}\right) \mathbf{n}+\nabla^{s} \cdot \boldsymbol{\tau}^{s}= & 8 \mu^{i} U \sin ^{2} \theta \sin 2 \varphi \mathbf{e}_{r} \\
& -4 \mu^{i}(U+2 V) \sin 2 \theta \sin 2 \varphi \mathbf{e}_{\theta} \\
& -8 \mu^{i}(U+2 V) \sin \theta \cos 2 \varphi \mathbf{e}_{\varphi} .
\end{aligned}
$$

\subsection{Evaluation of the bound}

In order to derive a closed-form bound, we must introduce (51), (53), (54) and (55) in (43). The evaluation of the corresponding integrals is rather involved, and will not be reproduced here. We find successively

$$
\begin{aligned}
&\left(\overline{\boldsymbol{\tau}}^{i}+\frac{3}{a} \overline{\boldsymbol{\tau}}^{s}\right): \mathbf{E}= 4 E\left[\left(\mu^{i}-\mu^{m}\right)\left(S-\frac{21}{5} \frac{T}{1-2 v^{i}}\right)\right. \\
&\left.+\frac{4}{5} \mu^{i}(U+6 V)\right], \\
& \frac{1}{\left|\Omega^{p}\right|} \int_{\mathbf{x} \in \Omega^{p}} \boldsymbol{\tau}^{i}(\mathbf{x}):\left(\mathbf{c}^{i}-\mathbf{c}^{m}\right)^{-1}: \boldsymbol{\tau}^{i}(\mathbf{x}) \mathrm{d}^{3} \mathbf{x}= \\
& 4\left(\mu^{i}-\mu^{m}\right)\left(S-\frac{21}{5} \frac{T}{1-2 v^{i}}\right)^{2}+\frac{252}{5}\left(\kappa^{i}-\kappa^{m}\right) T^{2} \\
&+\frac{8}{75}\left(\mu^{i}-\mu^{m}\right)\left[34+24 \frac{\kappa^{i}}{\mu^{i}}+171\left(\frac{\kappa^{i}}{\mu^{i}}\right)^{2}\right] T^{2}, \\
& \frac{a^{2}}{\left|\Omega^{p}\right|} \int_{|\mathbf{n}|=1} \tau^{s}(\mathbf{n}):\left(\mathbf{c}_{3 \mathrm{~d}}^{s}\right)^{-1}: \tau^{s}(\mathbf{n}) \mathrm{d}^{2} \mathbf{n}= \\
& \frac{64}{5} a\left(\mu^{i}\right)^{2}\left(\frac{U^{2}}{\kappa^{s}}+6 \frac{V^{2}}{\mu^{s}}\right),
\end{aligned}
$$




$$
\begin{array}{r}
\left(\overline{\boldsymbol{\tau}}^{i}+\frac{3}{a} \overline{\boldsymbol{\tau}}^{s}\right): \mathbf{P}^{\mathrm{sph}}:\left(\overline{\boldsymbol{\tau}}^{i}+\frac{3}{a} \overline{\boldsymbol{\tau}}^{s}\right)=\frac{24}{5} \frac{\kappa^{m}+2 \mu^{m}}{\mu^{m}\left(3 \kappa^{m}+4 \mu^{m}\right)} \\
{\left[\left(\mu^{i}-\mu^{m}\right)\left(S-\frac{21}{5} \frac{T}{1-2 \nu^{i}}\right)+\frac{4}{5} \mu^{i}(U+6 V)\right]^{2}}
\end{array}
$$

Evaluation of the self-influence term (32) is more involved. A prerequisite is the determination of the solution to the generalized inhomogeneity problem. Appendix A indicates how this solution is derived. Then (32) is evaluated; after lengthy calculations, simplification and reordering, the following result is found

$$
\begin{aligned}
-\frac{1}{\Omega^{p}} & \int_{\mathbf{x} \in \Omega^{p}} \boldsymbol{\tau}^{p}(\mathbf{x}): \boldsymbol{\varepsilon}^{p}(\mathbf{x}) \mathrm{d}^{3} \mathbf{x}= \\
& \frac{24}{5} \frac{\kappa^{m}+2 \mu^{m}}{\mu^{m}\left(3 \kappa^{m}+4 \mu^{m}\right)}\left[\left(\mu^{i}-\mu^{m}\right)\left(S-\frac{21}{5} \frac{T}{1-2 v^{i}}\right)\right. \\
& \left.+\frac{4}{5} \mu^{i}(U+6 V)\right]^{2} \\
& +\frac{12}{875} \frac{6 \kappa^{m}+17 \mu^{m}}{\mu^{m}\left(3 \kappa^{m}+4 \mu^{m}\right)}\left[\frac{\mu^{i}-\mu^{m}}{3 \mu^{i}}\left(57 \kappa^{i}+4 \mu^{i}\right) T\right. \\
& \left.-16 \mu^{i}(U+V)\right]^{2} \\
& +\frac{36}{25} \frac{\kappa^{m} \mu^{i}-\kappa^{i} \mu^{m}}{3 \kappa^{m}+4 \mu^{m}}[96 T(U+V) \\
& \left.-\left(105 \frac{\kappa^{i}-\kappa^{m}}{\mu^{i}}+\frac{\mu^{i}-\mu^{m}}{\mu^{i}} \frac{9 \kappa^{i}+8 \mu^{i}}{\mu^{i}}\right) T^{2}\right] .
\end{aligned}
$$

Substitution of (58)-(62) in (43) then leads to a lower bound on $\langle G\rangle E^{2}$ for any value of $E, S$, $T, U$ and $V$.

\subsection{Optimization of the bound}

For a given value of the macroscopic strain $E$, inequality (43) formally reads

$$
\begin{aligned}
\frac{1}{2} \mathbf{E}:\langle\mathbf{C}\rangle: \mathbf{E} \geq & \frac{1}{2} \mathbf{E}: \mathbf{c}^{m}: \mathbf{E}+f\left(\overline{\boldsymbol{\tau}}^{i}+\frac{3}{a} \overline{\boldsymbol{\tau}}^{s}\right): \mathbf{E} \\
& +\mathcal{H}(S, T, U, V),
\end{aligned}
$$

where $\mathcal{H}(S, T, U, V)$ is a quadratic form, which can be maximized with respect to its free parameters $S, T, U$ and $V$. At the stationary point of $\mathcal{H},(63)$ then reads

$$
\frac{1}{2} \mathbf{E}:\langle\mathbf{C}\rangle: \mathbf{E} \geq \frac{1}{2} \mathbf{E}: \mathbf{c}^{m}: \mathbf{E}+\frac{f}{2}\left(\overline{\boldsymbol{\tau}}^{i}+\frac{3}{a} \overline{\boldsymbol{\tau}}^{s}\right): \mathbf{E} .
$$

The stationary point of $\mathcal{H}$ linearly depends on the macroscopic strain $E$, and we note $E S^{*}$, $E T^{*}, E U^{*}$, and $E V^{*}$ the values of $S, T, U$ and $V$ at this point. Substituting (45) and (58), and simplifying by $E^{2}$ in (64), we get the lower bound on the effective shear modulus $\langle G\rangle$

$\langle G\rangle \geq \mu^{m}+f\left[\left(\mu^{i}-\mu^{m}\right)\left(S^{*}-\frac{21}{5} \frac{T^{*}}{1-2 v^{i}}\right)+\frac{4}{5} \mu^{i}\left(U^{*}+6 V^{*}\right)\right]$. 
Using (58)-(62), a closed-form for the above lower bound can be derived. After simplification and reordering, this bound reads as

$\langle G\rangle \geq \mu^{m}+\frac{P}{Q}$,

where

$$
\begin{aligned}
P= & 5 f \mu^{m}\left(3 \kappa^{m}+4 \mu^{m}\right)\left[M N+8\left(6 \kappa^{i}+17 \mu^{i}\right) \frac{\kappa^{s} \mu^{s}}{a^{2}}\right. \\
& \left.+7 \mu^{i}\left(3 \kappa^{i}+4 \mu^{i}\right) \frac{6 \kappa^{s}+\mu^{s}}{a}\right],
\end{aligned}
$$

and

$$
\begin{aligned}
Q= & {\left[5 \mu^{m}\left(3 \kappa^{m}+4 \mu^{m}\right)+6(1-f)\left(\kappa^{m}+2 \mu^{m}\right) N\right] M } \\
& +8\left(6 \kappa^{i}+17 \mu^{i}\right)\left[\mu^{m}\left(3 \kappa^{m}+4 \mu^{m}\right) \frac{6 \kappa^{s}+\mu^{s}}{a}\right. \\
& \left.+6(1-f)\left(\kappa^{m}+2 \mu^{m}\right) \frac{\kappa^{s} \mu^{s}}{a^{2}}\right] \\
& +42(1-f) \mu^{i}\left(\kappa^{m}+2 \mu^{m}\right)\left(3 \kappa^{i}+4 \mu^{i}\right) \frac{6 \kappa^{s}+\mu^{s}}{a},
\end{aligned}
$$

with

$$
\begin{aligned}
& M=57 \kappa^{i} \mu^{i}+48 \kappa^{i} \mu^{m}+4\left(\mu^{i}\right)^{2}+136 \mu^{i} \mu^{m}, \\
& N=\mu^{i}-\mu^{m}+\frac{\mu^{s}-\kappa^{s}}{a} .
\end{aligned}
$$

When interface effects are disregarded (that is $\kappa^{s}=0, \mu^{s}=0$ ), the classical Hashin and Shtrikman [4] bound is retrieved. When $\kappa^{s} \neq 0$, and $\mu^{s} \neq 0$, the proposed bound can be proved to coincide with the Mori-Tanaka estimate of Duan et al. [1]. As already mentioned in section 1.1 , our definition of $\kappa^{s}$ differs by a factor 2 from the definition adopted by these authors.

In a previous paper [6], we have already proved that the Mori-Tanaka estimate of the effective bulk modulus of a nanocomposite coincides with the corresponding Hashin-Shtrikman lower bound. The present study extends this result to the effective shear modulus. In turn, those two results generalize the well-known identity between Mori-Tanaka estimates and Hashin-Shtrikman bounds for statistically isotropic composites without surface effects.

Finally, it should be noted that calculations not reproduced here show that neither of the optimum values $S^{*}, T^{*}, U^{*}, V^{*}$ are identically null. This means that the apparent complexity of the polarization fields selected in this calculation, see (52), (53), (54) and (55), was in fact necessary in order to derive the desired bound.

\section{Conclusion}

In this paper, we apply our previously introduced variational framework for nanocomposites, in which surface stresses can arise, to the derivation of bounds on the shear modulus. The technique draws on the polarization methods developed by Willis [10], and generalized by Bornert 
et al. [7]. In a recent paper, Le Quang and He [3] had questioned the possibility to derive secondorder bounds for nanocomposites; the present work provides an affirmative answer to this question.

The technique is specifically applied to nanocomposites with monosized, spherical particles. It is shown that the resulting Hashin-Shtrikman type lower-bound on the shear modulus coincides with previously known [1] Mori-Tanaka estimates. Two assumptions were made: $i$. the composite is statistically homogeneous and isotropic, and $i i$. the stiffness of the interface is positive definite.

While assumption $i$. is classical, assumption $i$. may fail to be true. Indeed, through numerical simulation, Shenoy [5] has exhibited a composite for which the stiffness of the interface is not positive definite. In this case, (66) no longer holds; however, our result can still be considered as an estimate.

The results proposed here were developed in the context of linear elasticity. They can readily be generalized to other classes of problems, such as conduction/thermal diffusion. Extension of these result to non-linear mechanics could also be considered, using the variational frameworks proposed by eg Ponte Castañeda [15], Willis [16].

\section{Appendix A. Solution to the generalized inhomogenity problem}

In this section, we seek the solution to the problem of a unique spherical elastic inhomogeneity submitted to the polarization field $\tau^{p}$ defined by (37), (51), (53), (54) and (55). In other words, we seek the solution to the problem defined by equations (30) and (31). It is shown here that $\mathbf{u}$ can be conveniently written in terms of Love's solution, see equations (46) to (48), and four dimensionless constants $A_{\mathrm{int}}, B_{\mathrm{int}}, C_{\mathrm{ext}}$ and $D_{\mathrm{ext}}$. Outside the inhomogeneity (for $|\mathbf{x}|>a$ ), the following form is adopted

$\mathbf{u}(\mathbf{x})=\mathbf{u}_{\mathrm{ext}}(\mathbf{x})=\mathcal{U}\left[v^{m}, 0,0, a^{5} C_{\mathrm{ext}}, a^{3} D_{\mathrm{ext}}\right](\mathbf{x})$.

Equation (31) is obviously satisfied, while (49) shows that (30) holds for $|\mathbf{x}|>a$ (since $\tau^{p} \equiv 0$ outside the inhomogeneity). Inside the inhomogeneity (for $|\mathbf{x}|<a),($ A.1) is not a suitable choice for the displacement field; instead, it will be shown that the solution takes the following form

$$
\begin{aligned}
\mathbf{u}(\mathbf{x})=\mathbf{u}_{\text {int }}(\mathbf{x})= & \mathcal{U}\left[v^{m}, A_{\text {int }}, B_{\text {int }} / a^{2}, 0,0\right](\mathbf{x}) \\
& +\mathcal{U}\left[v^{i}, S, T / a^{2}, 0,0\right](\mathbf{x}) .
\end{aligned}
$$

Then, from (51), we have for $|\mathbf{x}|<a$

$$
\begin{aligned}
\mathbf{c}^{m}: \boldsymbol{\varepsilon}_{\text {int }}(\mathbf{x})+\boldsymbol{\tau}^{p}(\mathbf{x})= & \mathbf{c}^{m}: \mathcal{E}\left[v^{m}, A_{\text {int }}, B_{\text {int }} / a^{2}, 0,0\right](\mathbf{x}) \\
& +\mathbf{c}^{i}: \mathcal{E}\left[v^{i}, S, T / a^{2}, 0,0\right](\mathbf{x}) .
\end{aligned}
$$

Observing that in the right-hand side, each occurence of the Love strains $\mathcal{E}$ are evaluated for the same value of the Poisson ratio as the stiffness tensor with whom they are contracted, application of (49) again shows that (30) is satisfied inside the inhomogeneity. For (A.1)-(A.2) to be the solution to the generalized inhomogeneity problem (30)-(31), two requirements remain to be satisfied, namely $i$. u must be continuous across the interface $|\mathbf{x}|=a$, and ii. the jump conditions (3)-(5) must be enforced.

In the remainder of this section, it is shown that $i$. and $i i$. lead to four independent linear equations in the unknowns $A_{\text {int }}, B_{\text {int }}, C_{\text {ext }}$ and $D_{\text {ext }}$, the solution of which is however too complicated to be presented here. 
Continuity of the displacement. This requirement results in two independent equations (continuity of $u_{\theta}$ and $u_{\varphi}$ lead to the same equation)

$$
\begin{aligned}
& A_{\text {int }}-\frac{6 v^{m}}{1-2 v^{m}} B_{\text {int }}-3 C_{\mathrm{ext}}-\frac{5-4 v^{m}}{1-2 v^{m}} D_{\mathrm{ext}}=-S+\frac{6 v^{i}}{1-2 v^{i}} T, \\
& A_{\mathrm{int}}-\frac{7-4 v^{m}}{1-2 v^{m}} B_{\mathrm{int}}+2 C_{\mathrm{ext}}-2 D_{\mathrm{ext}}=-S+\frac{7-4 v^{i}}{1-2 v^{i}} T .
\end{aligned}
$$

Equilibrium of the interface. The tangential equilibrium equations being also redundant, equilibrium of the interface (3)-(5) leads to only two independent equations

$$
\begin{aligned}
& \mu^{m}\left(A_{\mathrm{int}}+\frac{3 v^{m}}{1-2 v^{m}} B_{\mathrm{int}}+12 C_{\mathrm{ext}}+2 \frac{5-v^{m}}{1-2 v^{m}} D_{\mathrm{ext}}\right)= \\
& -\mu^{i}\left(S+\frac{3 v^{i}}{1-2 v^{i}} T-4 U\right),
\end{aligned}
$$

and

$$
\begin{gathered}
\mu^{m}\left(-A_{\mathrm{int}}+\frac{7+2 v^{m}}{1-2 v^{m}} B_{\mathrm{int}}+8 C_{\mathrm{ext}}+2 \frac{1+v^{m}}{1-2 v^{m}} D_{\mathrm{ext}}\right)= \\
\mu^{i}\left(S-\frac{7+2 v^{i}}{1-2 v^{i}} T+4 U+8 V\right) .
\end{gathered}
$$

Determination of the solution. It can be shown that the system (A.4)-(A.7) is non-singular. Its solution provides the values of the integration constants $A_{\mathrm{int}}, B_{\mathrm{int}}, C_{\mathrm{ext}}$ and $D_{\mathrm{ext}}$. The solution to the polarized problem is then found after substitution of these values into expressions (A.1) and (A.2). Subsequent derivation provides the expression of the strains $\boldsymbol{\varepsilon}^{p}$.

\section{References}

[1] H. L. Duan, J. Wang, Z. P. Huang, B. L. Karihaloo, Size-dependent effective elastic constants of solids containing nano-inhomogeneities with interface stress, Journal of the Mechanics and Physics of Solids 53 (7) (2005) 1574 1596.

[2] H. Le Quang, Q.-C. He, Size-dependent effective thermoelastic properties of nanocomposites with spherically anisotropic phases, Journal of the Mechanics and Physics of Solids 55 (9) (2007) 1899-1931.

[3] H. Le Quang, Q. C. He, Variational principles and bounds for elastic inhomogeneous materials with coherent imperfect interfaces, Mechanics of Materials 40 (10) (2008) 865-884.

[4] Z. Hashin, S. Shtrikman, On some variational principles in anisotropic and nonhomogeneous elasticity, Journal of the Mechanics and Physics of Solids 10 (4) (1962) 335-342.

[5] V. B. Shenoy, Atomistic calculations of elastic properties of metallic fcc crystal surfaces, Physical Review B 71 (9) (2005) 1-11.

[6] S. Brisard, L. Dormieux, D. Kondo, Hashin-Shtrikman bounds on the bulk modulus of a nanocomposite with spherical inclusions and interface effects, Computational Materials Science 48 (3) (2010) 589-596.

[7] M. Bornert, C. Stolz, A. Zaoui, Morphologically representative pattern-based bounding in elasticity, Journal of the Mechanics and Physics of Solids 44 (3) (1996) 307-331.

[8] Y. Z. Povstenko, Theoretical investigation of phenomena caused by heterogeneous surface-tension in solids, Journal of the Mechanics and Physics of Solids 41 (9) (1993) 1499-1514.

[9] P. Müller, A. Saul, Elastic effects on surface physics, Surface Science Reports 54 (5-8) (2004) 157-258

[10] J. R. Willis, Bounds and self-consistent estimates for the overall properties of anisotropic composites, Journal of the Mechanics and Physics of Solids 25 (3) (1977) 185-202.

[11] P. Ponte Castañeda, J. R. Willis, The effect of spatial distribution on the effective behavior of composite materials and cracked media, Journal of the Mechanics and Physics of Solids 43 (12) (1995) 1919-1951. 
[12] E. Kröner, Bounds for effective elastic moduli of disordered materials, Journal of the Mechanics and Physics of Solids 25 (2) (1977) 137-155.

[13] A. E. Love, Treatise on the mathematical theory of elasticity, Dover Publications, 1927.

[14] E. Hervé, A. Zaoui, N-layered inclusion-based micromechanical modeling, International Journal of Engineering Science 31 (1) (1993) 1-10.

[15] P. Ponte Castañeda, The effective mechanical properties of nonlinear isotropic composites, Journal of the Mechanics and Physics of Solids 39 (1) (1991) 45-71.

[16] J. R. Willis, On methods for bounding the overall properties of nonlinear composites, Journal of the Mechanics and Physics of Solids 39 (1) (1991) 73-86. 